\title{
Frequency of Neonatal Hypocalcaemia and Its Correlation with Risk Factors
}

Ines Bošnjak ${ }^{1}$ and Marjana Jerković Raguž ${ }^{\star *}$

${ }^{1}$ Faculty of Medicine University of Mostar, Bosnia and Herzegovina

${ }^{2}$ Department of Neonatology and Intensive Care Unit, Clinic for Children's Diseases, University Clinical Hospital of Mostar, Bosnia and Herzegovina

*Corresponding author: Raguž MJ, Department of Neonatology and Intensive Care Unit, Clinic for Children's Diseases, University Clinical Hospital of Mostar, Bosnia and Herzegovina; E-mail: marjanajerkovic@yahoo.co.uk

Received date: September 12, 2017; Accepted date: October 6, 2017; Published date: October 10, 2017

Copyright: $\odot 2017$ Bošnjak I, et al. This is an open-access article distributed under the terms of the Creative Commons Attribution License, which permits unrestricted use, distribution, and reproduction in any medium, provided the original author and source are credited.

\begin{abstract}
Aim: To determine the frequency of hypocalcaemia in newborns treated at the Neonatology Department of the Children's Disease of the University Clinical Hospital in Mostar and to examine the correlation with certain risk factors of the mother and the newborn

Examinees and methods: The study included 98 newborns treated at the Department of Neonatology and Intensive Treatment of newborns of the Children's Diseases of the University Clinical Hospital in Mostar in one year period (2016). The parameters of newborns (gender, birth weight, gestational age, the age of the newborn at the time of occurrence of hypocalcaemia, lowest serum calcium levels, IUGR, pathological conditions: asphyxia, jaundice, sepsis, perinatal infection, RDS, urinary tract infection, other mineral dysbalances (Mg, Na, Glucose), and mother (age, type of birth, medication, illness: hypertension, diabetes, infection before delivery) were observed.
\end{abstract}

Result: Out of a total of 272 newborns treated at the Neonatology Department, hypocalcaemia was found in 98 subjects (36\%), while the incidence of hypocalcaemia in 1831 live-born infants in 2016 was $18.6 \%$.

The most significant and the most frequent risk factors of mothers include parity, age, type of birth and use of medication, especially antibiotics, during pregnancy. Pregnant women with first delivery, aged between 28 and 39 , who have given birth naturally and who used the medicines during pregnancy are the most prone of giving birth to newborns with hypocalcaemia.

Gender, gestational age, and birth weight of newborns have been shown to be significant and common risk factors for the development of hypocalcaemia. Male term newborns weighing between 2501 and $3500 \mathrm{~g}$ have the greatest predisposition to develop this condition. There were more premature infants in relation to the term infants, however, newborns were observed through two gestational groups, so it was not highlighted on whole sample. Among the subjects, there were more newborns with early hypocalcaemia diagnosis than the late ones. The most common age of the newborn at the time of hypocalcaemia occurrence is within the first $24 \mathrm{~h}$ of life. The most commonly associated pathological conditions were jaundice, perinatal infection and hypoglycemia, which was not statistically significant.

Conclusion: Hypocalcaemia is an important clinical sign that we often do not think about and life-long is important. Therefore, more attention should be devoted to identifying risk factors, prevention, early identification and metabolic support for sick newborns.

Keywords: Newborn; Calcium; Hypocalcaemia; Risk factors

\section{Introduction}

Hypocalcaemia is a laboratory and clinical abnormality that is observed with relative frequency, especially in the newborn age. Although neonatal hypocalcaemia can be a potentially life-threatening condition, laboratory hypocalcaemia is often asymptomatic and transient. Moreover, prevalence varies by gestational age (GA) and perinatal disease. For these reasons, the definition of hypocalcaemia in the neonatal population is not well established, and the therapeutic cut off level of low serum calcium is controversial [1].
Total serum calcium levels of less than $2.2 \mathrm{mmol} / \mathrm{L}$ and ionized calcium levels of less than $1.2 \mathrm{mmol} / \mathrm{L}$ are considered hypocalcaemia [2].

The etiology of hypocalcaemia varies with the time of onset and the associated illnesses of the child [2]. Overall, one of the most common causes of hypocalcaemia in children is renal failure, which results in hypocalcaemia because of inadequate 1-hydroxylation of 25hydroxyvitamin $\mathrm{D}$ and hypophosphatemia due to diminished glomerular filtration. The causes of hypocalcaemia can be classified by the child's age at presentation [3].

Early onset hypocalcaemia occurs in the first 3 days of life and is associated with prematurity, infants of diabetic mothers, intrauterine growth restriction, and perinatal asphyxia. 
Late onset hypocalcaemia occurs after the first 3 days of life because of hyperparathyroidism, the feeding of high phosphate formula, DiGeorge syndrome (chromosome 22q11.2 deletion), hypomagnesaemia and vitamin D deficiency [4].

The neonate with hypocalcaemia may be asymptomatic; the less mature the infant, the more subtle and varied are the clinical manifestations. In the neonatal period, the main clinical signs of hypocalcaemia are jitteriness (increased neuromuscular irritability and activity) and generalized convulsions [5].

Hypocalcaemia can be manifested with nonspecific symptoms, such as poor sucking, vomiting, and somnolence [6]. Infants may also be lethargic, eat poorly, vomit, and have abdominal distention [5].

Hypocalcaemia has an important significance and place in a wide spectrum of diseases that occur specifically in the newborn age. Because of the unspecified clinical presentation and not observing hypocalcaemia as a unique diagnosis, there is a danger that it will be overlooked. It is necessary to pay attention to the importance of calcium disorders in newborns and explore risk factors as the recognition of risk is the first step toward diagnosis.

\section{Aim}

The aim was to determine the frequency of hypocalcaemia in newborns treated at the Neonatology Department of the Children's Disease of the University Clinical Hospital in Mostar and to examine the correlation with certain risk factors of the mother and the newborn.

\section{Examinees and Methods}

Retrospective epidemiological research included all newborns, 98 of them who had calcium concentrations less than $2.20 \mathrm{mmol} / \mathrm{L}$ in their serum. Those newborns were treated at the Department of Neonatology and Intensive Treatment of newborns of the Children's Diseases of the University Clinical Hospital in Mostar, some of them were moved from maternity clinic and some of them came from home to admission.

In this study all newborns with diagnosed hyperparathyroidism (e.g due to DiGeorge syndrome with agenesis or parathyroid gland dysgenesis) and newborns that were treated at the Department of Gynecology and Obstetrics, Department of Neonatology, and were not treated at the Neonatology Department of the Pediatric Disease Clinic SKB Mostar, were excluded.

The parameters of newborns (gender, weight, gestational age, the age of the newborn at the time of occurrence of hypocalcaemia, lowest serum calcium levels, IUGR, pathological conditions: asphyxia, jaundice, sepsis, perinatal infection, RDS, urinary tract infection, other mineral dysbalances (Mg, Na, GUK)) and mother (age, type of birth, medication, illness: hypertension, diabetes, an infection before delivery) were observed.

The data were collected by examining the medical records, medical histories, discharge summary of the newborns, as well as examining relocation lists of mothers and children. During the research, data from the socio-biographical and gynecological-obstetrician questionnaires were used, which were taken from each pregnant woman at hospital admissions.
For the test of statistical significance Chi-square $\left(\chi^{2}\right)$ test and Fisher's exact test were used. The probability level of $p<0.05$ was taken as statistically significant.

\section{Results}

In the period from 01.01.2016 to 01.01.2017, the number of liveborn infants was 1821 .

272 newborns were treated at the Department of Neonatology and Intensive Treatment of newborns of the Children's Diseases of the University Clinical Hospital in Mostar.

Out of a total of 272 newborns treated at the Neonatology Department, hypocalcaemia was found in 98 subjects (36\%), while the incidence of hypocalcaemia in 1831 live-born infants in 2016 was $18.6 \%$.

\begin{tabular}{|l|l|l|l|l|}
\hline Risk factors of the newborn & $\mathbf{N}$ & $\%$ & $\mathbf{X}^{2}$ & $\mathbf{p}$ \\
\hline Gender & & & 4.939 & 0.026 \\
\hline M & 60 & 61.2 & & \\
\hline F & 38 & 38.8 & & \\
\hline$<33$ weeks & & & 9.143 & 0.010 \\
\hline $34-36$ weeks & 30 & 30.6 & & \\
\hline$>37$ weeks & 22 & 22.4 & & \\
\hline Birth weight & 46 & 46.9 & & \\
\hline$<1000 \mathrm{~g}$ & & & 36.49 & $<0.001$ \\
\hline $1001-1500 \mathrm{~g}$ & 0 & 0 & & \\
\hline $1501-2500 \mathrm{~g}$ & 5 & 5.1 & & \\
\hline $2501-3500 \mathrm{~g}$ & 38 & 38.8 & & \\
\hline $3500-4000 \mathrm{~g}$ & 40 & 40.8 & & \\
\hline
\end{tabular}

Table 1: Representation of hypocalcaemia according to gender, gestational age and birth weight of the newborn.

Results show that more newborns were male $\left(\chi^{2}=4.939 ; \mathrm{p}=0.026\right)$, gestation $>37$ weeks $\left(\chi^{2}=9.143 ; \mathrm{p}=0.010\right)$ and birth weight between 2501 and 3500 g. $\left(\chi^{2}=36.49 ; p<0.001\right)$. By testing, it was found that this was a statistically significant difference for all three parameters. 
Page 3 of 4

\section{The age of the newborn at the time of occurrence of hypocalcaemia}

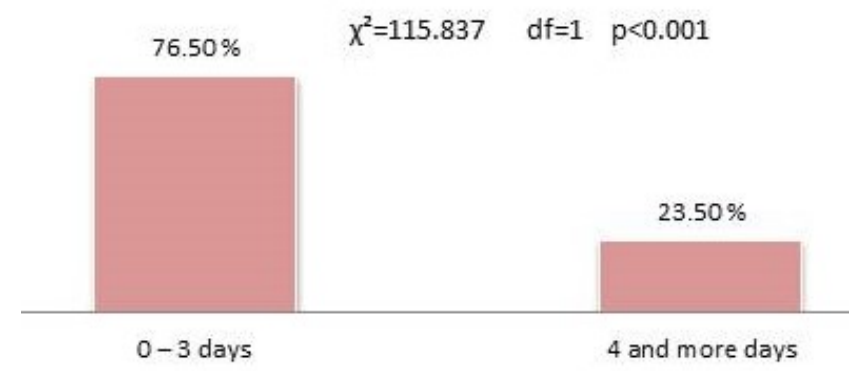

Figure 1: Representation of hypocalcaemia according to the age of the newborn at the time of occurrence of hypocalcaemia.

The analysis found that more infants with low calcium concentrations were observed within the first three days of life, which was statistically significant. We notice that more newborns were diagnosed with early onset hypocalcaemia $(76.50 \%)$ compared to late onset hypocalcaemia $(23.50 \%) \quad\left(\chi^{2}=115.837 ; \mathrm{p}<0.001\right)$. The most common age of the newborn at the time of occurrence of hypocalcaemia is within the first $24 \mathrm{~h}$ of life $(\mathrm{Mo}=0)$.

\begin{tabular}{|l|l|l|l|l|}
\hline $\begin{array}{l}\text { Associated } \\
\text { pathological } \\
\text { conditions }\end{array}$ & $\mathbf{N}$ & $\%$ & $\mathbf{X}^{\mathbf{2}}$ & $\mathbf{p}$ \\
\hline Asphyxia & 22 & 22.4 & 29.755 & $<0.001$ \\
\hline Jaundice & 40 & 40.8 & 62.082 & $<0.001$ \\
\hline Sepsis & 9 & 9.2 & 65.306 & $<0.001$ \\
\hline Perinatal infection & 35 & 35.7 & 8.000 & 0.005 \\
\hline RDS & 22 & 22.4 & 29.755 & $<0.001$ \\
\hline IUGR & 10 & 10.2 & 62.082 & $<0.001$ \\
\hline Hypoglycaemia & 43 & 43.9 & 1.469 & 0.225 \\
\hline Hypomagnesaemia & 11 & 11.2 & 58.939 & $<0.001$ \\
\hline Hyponatremia & 21 & 21.4 & 32.000 & $<0.001$ \\
\hline Urinary tract infection & 17 & 17.3 & 41.796 & $<0.001$ \\
\hline
\end{tabular}

Table 2: Representation of hypocalcaemia according to associated pathological conditions.

The most frequent conditions were jaundice, perinatal infection and hypoglycemia. Statistically, it was found that more newborns had no associated pathological conditions $(\mathrm{p}<0.001)$.

Mothers of tested newborns more often were nulliparous $\left(\chi^{2}=34.735 ; \mathrm{p}<0.001\right)$, aged $28-39$ years $\left(\chi^{2}=61.837 ; \mathrm{p}<0.001\right)$, who gave birth naturally $\left(\chi^{2}=44.163 ; \mathrm{p}<0.001\right)$.
The diseases of the mother, that we observed, were not so common $(\mathrm{p}<0.001)$. Most of the mothers took some type of medication during pregnancy $\left(\chi^{2}=19.755 ; \mathrm{p}<0.001\right)$. All this results are shown as a statistically significant information, except the consumption of antibiotics during pregnancy $(\mathrm{p}=0.187)$.

\begin{tabular}{|c|c|c|c|c|}
\hline Maternal risk factors & $\mathbf{N}$ & $\%$ & $x^{2}$ & $\mathbf{p}$ \\
\hline Mother's age & & & 61.837 & $<0.001$ \\
\hline$<20$ & 3 & 3.1 & & \\
\hline $21-28$ & 35 & 35.7 & & \\
\hline $29-38$ & 51 & 52.0 & & \\
\hline$>30$ & 9 & 9.2 & & \\
\hline Parity & & & 34.735 & $<0.001$ \\
\hline First pregnancy & 44 & 44.9 & & \\
\hline Second pregnancy & 32 & 32.7 & & \\
\hline Third pregnancy & 16 & 16.3 & & \\
\hline $\begin{array}{l}\text { Fourth and more } \\
\text { pregnancy }\end{array}$ & 6 & 6.1 & & \\
\hline Type of birth & & & 44.163 & $<0.001$ \\
\hline Vaginal birth & 52 & 53.1 & & \\
\hline Caesarean section & 44 & 44.9 & & \\
\hline Vacuum & 2 & 2 & & \\
\hline Diabetes & 14 & 14.3 & 50.000 & $<0.001$ \\
\hline Hypertension & 8 & 8.2 & 68.612 & $<0.001$ \\
\hline Infection before delivery & 19 & 19.4 & 36.735 & $<0.001$ \\
\hline Other illnesses & 30 & 30.6 & 14.735 & $<0.001$ \\
\hline $\begin{array}{l}\text { Medication } \\
\text { consummation }\end{array}$ & 71 & 72.4 & 19.755 & $<0.001$ \\
\hline $\begin{array}{l}\text { Antibiotics } \\
\text { consummation }\end{array}$ & 42 & 42.9 & 1.742 & 0.187 \\
\hline
\end{tabular}

Table 3: Representation of hypocalcaemia in newborn according to maternal risk factors.

\section{Discussion}

The research we conducted for a one-year period, during 2016, showed a significant incidence of hypocalcaemia in the newborn age. Out of a total of 272 newborns treated at the Neonatology Department, hypocalcaemia was found in 98 subjects (36\%), while the incidence of hypocalcaemia in 1831 live-born infants in 2016 was $18.6 \%$.

This research has shown that hypocalcaemia is more common with the observed risk factors. As the most important and most common risk factors for newborns, gender, gestational age and birth weight were specified. Most common maternal risk factors were age, parity, type of birth and taking medication during pregnancy.

Regarding the risk factors of newborns involved in the study, our research has shown that a greater number of newborns had male gender and birth weight between 2501 and $3500 \mathrm{~g}$, which was 
statistically significant and in correlation with the research in 2015, which had the goal to describe the clinical characteristics of hypocalcaemia and to show the risk factors associated with the development of the hypocalcaemia. It included 53 newborns born in 2015, in South Korea [1]. Our research showed that incidence rates were higher in newborn with gestational age $>37$ weeks, although overall there were more premature infants (gestational age $<37$ weeks), which is consistent with literature [4].

It was observed that more newborns were diagnosed with early onset hypocalcaemia compared to late onset hypocalcaemia, and this result is consistent with the above-mentioned research in which hypocalcaemia in newborns appeared within the first three days of life [1]. According to this same study, the most common age of hypocalcaemia was the third day, while our research showed that hypocalcaemia is most common in the first $24 \mathrm{~h}$ of life. Our research has not shown correlation or statistical significance between associated pathological conditions and reduced calcium serum levels, although literature suggests that hypocalcaemia is most common with asphyxia and IUGR [4]. In those newborns that had asphyxia, RDS and perinatal infection statistically significantly hypocalcaemia was developed in early form. Asphyxia as a risk factor in the study from South Korea was present, but not in substantial percentage (13.2\%) [7].

Mothers of tested newborns more often were nulliparous, aged 28-39 years, who gave birth naturally. The diseases of the mother, which we observed, diabetes, hypertension, and prenatal infections did not appear to be statistically significant, while most mothers took some type of medication during pregnancy.

In literature, diabetes is shown as a major risk factor for mothers and we have obtained almost the same result as the result from South Korean research (about 14\% of mothers was affected by diabetes) [1]. No similar research was found for other observed risk factors of the mother.

Hypocalcaemia is an important clinical sign that we often do not think about and life-long is important. Therefore, more attention should be devoted to identifying risk factors, prevention, early identification and metabolic support for sick newborns.

This research has shown that hypocalcaemia is an important clinical sign of severe conditions and how important it is to identify risk factors and respond timely to prevent the consequences. If we were to discuss possible deficiency of this research, it would certainly be the number of examinees and the time period of the research. For this reason, future researches on this topic should certainly be carried out on a larger sample and researches should be expanded for a longer period of time. Also, in the future, it would be desirable to include some other parameters, which would be considered as risk factors for the emergence of hypocalcaemia in newborns, such as nutrition of newborns, vitamin D and phosphate concentrations in serum of mothers and newborns, in order to obtain even clearer and better perception of this issue. During the work and the search of relevant literature, there was a lack of research on this and similar topics, so it was not possible to compare the results of this research at all levels with some of the already existing researches. However, this study is significant because it has provided a specific description of the hypocalcaemia characteristics, which provides valuable information and can help in understanding the mechanisms of newborn hypocalcaemia.

\section{Conclusion}

In 2016, the incidence of hypocalcaemia in 1831 live-born children was $18.6 \%$. Out of a total of 272 newborns treated at the Neonatology Department, hypocalcaemia was found in 98 subjects (36\%). As the most important and most common risk factors of the newborns, gender, gestational age and birth weight were specified. Most common maternal risk factors were age, parity, type of birth and taking medication during pregnancy. It was observed that more newborns were diagnosed with early onset hypocalcaemia compared to late onset hypocalcaemia. The most common age of hypocalcaemia was in the first $24 \mathrm{~h}$ of life.

\section{Conflict of Interest}

There is no any financial or other conflict of interest.

\section{References}

1. Cho WI, Yu HW, Chung HR, Shin CH, Yang SW, et al. (2015) Clinical and laboratory characteristics of neonatal hypocalcaemia. Ann Pediatr Endocrinol Metab 20: 86-91.

2. Gowen CW (2015) Fetal and Neonatal Medicine. In: Marcdante KJ, Kliegman RM (eds) Nelson Essentials of Pediatrics. Elsevier Saunders, Philadelphia.

3. Uhing MR, Kliegman RM (2013) Glucose, Calcium and Magnesium. In: Fanaroff AA, Fanaroff JM (eds) Klaus and Fanaroff's Care of the HighRisk Neonate. Elsevier Saunders, Philadelphia.

4. Sansevere AJ, Bergin AM (2017) Neonatal Seizures. In: Eichenwald EC, Hansen AR, Martin CR, Stark AR (eds) Cloherty and Stark's Manual of Neonatal Care, Philadelphia.

5. Abrams SA, Tiosano D (2015) Disorders of Calcium, Phosphorus, and Magnesium Metabolism in the Neonate. In: Martin RJ, Fanaroff AA, Walsh MC (eds) Fanaroff and Martin's Neonatal-Perinatal Medicine, Philadelphia.

6. Mardešić D (2003) Water, electrolyte, mineral disorders and acid-base disorders. In: Mardešić D and associates, Pediatrics, Zagreb.

7. Song JY, Shin YL, Yoo HW (2002) Clinical characteristics of symptomatic hypocalcemic infants. J Korean Soc Pediatr Endocrinol 7: 95-104. 